INRA Prod. Anim.,

2008, 21 (4), 361-366

\section{Mesure des nuisances olfactives associées à l'élevage porcin}

\author{
J.-L. BERDAGUÉ 1 , M. BONNEAU2
}

${ }^{1}$ INRA, UR 370 Qualité des produits animaux, F-63122 Saint-Genès Champanelle, France

2 INRA, UMR 1079 Systèmes d'Elevage, Nutrition Animale et Humaine, F-35590 Saint-Gilles, France

Courriel : Michel.Bonneau@rennes.inra.fr
Les nuisances olfactives sont, dans l'esprit du public, fortement associées à l'élevage porcin. Beaucoup de professionnels identifient ces nuisances comme l'un des, voire le facteur(s) limitant essentiel(s) de son développement ou même de son maintien. Ces nuisances peuvent être à l'origine de conflits de voisinage et provoquent immédiatement une levée de boucliers à l'encontre de tout projet d'extension ou d'établissement d'un élevage de porc.

Le projet «Porcherie verte» a voulu concentrer ses efforts sur les mises au point méthodologiques, et ceci pour deux raisons :

- les travaux antérieurs ont mis en évidence de nombreux composés malodorants, dont l'ammoniac, dans les effluents porcins. Il n'a cependant pas été possible d'identifier une liste courte de contributeurs majeurs. L'une des hypothèses est que ces contributeurs majeurs sont des composés extrêmement malodorants, présents à des concentrations très faibles qui n'autorisent pas leur détection par les méthodes d'analyse classiques. Les progrès récents des méthodes analytiques de séparation et d'identification autorisent de grands espoirs de pouvoir enfin les identifier. Ceci permettrait d'avoir une approche plus efficace de contrôle des nuisances olfactives. Les approches «boîte noire», seules possibles jusqu'à présent, sont très difficiles car la mesure des odeurs est longue et délicate, donc extrêmement coûteuse ;

- la difficulté de mesurer les odeurs (lourdeur, coût, subjectivité) est un réel handicap dans la gestion d'un problème qui entre souvent dans le domaine du passionnel, car posé dans un contexte conflictuel. La mise au point de méthodes rapides, simples et peu onéreuses de caractérisation qualitative et quantitative des odeurs faciliterait grandement l'objectivation d'un débat qui en a bien besoin.

\section{1 / Mises au point méthodo- logiques pour la caractéri- sation des odeurs de por- cherie}

La caractérisation des effluents volatils odorants issus des bâtiments d'élevage peut s'aborder par une approche sensorielle (utilisant un jury sur site ou en laboratoire) ou par une approche instrumentale.

L'approche sensorielle directe sur site connait de nombreuses limitations techniques et financières (déplacement d'un jury sur place, dépendance vis-àvis des conditions climatiques, saturation rapide des juges dans une atmosphère trop fortement odorante). La mesure normalisée du seuil de dilution olfactif à partir d'un prélèvement d'atmosphère dans une baudruche (normes AFNOR NF X 43-10, NF X 43103 and NF $X$ 43-104) néglige la dimension qualitative des odeurs et génère des artefacts analytiques liés à la condensation de l'eau et à l'adsorption des composés organiques volatils sur les parois des sacs de prélèvements.

Les approches instrumentales ont été, jusqu'à ce jour, plutôt décevantes. Elles se sont pour l'instant limitées à la chromatographie en phase gazeuse couplée à la spectrométrie de masse à des fins d'identification des composés organiques volatils, une technique lourde qui n'est pas envisageable pour un contrôle de routine des atmosphères. La Micro Extraction en Phase Solide (SPME) est une technique récente, simple et rapide de piégeage des composés organiques volatils, qui a déjà été utilisée pour la surveillance environnementale. Couplée à la Chromatographie en Phase Gazeuse-Spectrométrie de Masse (SPME-CPG-MS) elle permet d'obtenir une information détaillée sur la fraction volatile des matrices ou des atmosphères. Couplée directement à la Spectrométrie de Masse, sans séparation chromatographique préalable (SPME-MS), elle permet d'obtenir rapidement des spectres de masse caractéristiques de la composition globale des composés organiques volatils.

Le travail réalisé dans le cadre du programme «Porcherie verte» a consisté à mettre en place deux procédures de caractérisation des atmosphères de porcherie simples et efficaces. La première consiste en une analyse sensorielle délocalisée obtenue en piégeant les composés volatils des bâtiments d'élevage dans une phase lipidique dont les caractéristiques odorantes sont ensuite analysées en laboratoire par un jury entraîné. La seconde consiste à obtenir rapidement des signatures instrumentales des atmosphères de porcherie par SPME-MS. Une mise en relation des résultats analytiques obtenus par ces deux procédures de caractérisation des atmosphères a ensuite été tentée.

\section{1 / Matériel utilisé}

Les prélèvements de composés organiques volatiles ont été effectués dans 42 porcheries représentant la grande diversité des situations d'élevage existantes, en termes de stade physiologique des animaux (truies allaitantes, porcelets en post-sevrage ou porcs en engraissement) et de type de sol dans les bâtiments (caillebotis ou litière). Tous les prélèvements ont été effectués par le même expérimentateur qui a également procédé à une estimation de l'intensité odorante sur une échelle de 1 (très faible) à 10 (très odorant), qui s'est avérée très différente selon les situations (figure 1). Pour chaque porcherie, les prélèvements destinés aux analyses sensorielles (sur phase lipidique coulée dans une boîte de Pétri) et aux analyses instrumentales (sur fibres SPME) ont été réalisés simultanément à l'intérieur du bâtiment. La durée d'exposition était de deux heures. 
Figure 1. Intensité de l'odeur perçue sur site par l'expérimentateur en fonction de la situation d'élevage (d'après Bégnaud et al 2004a).

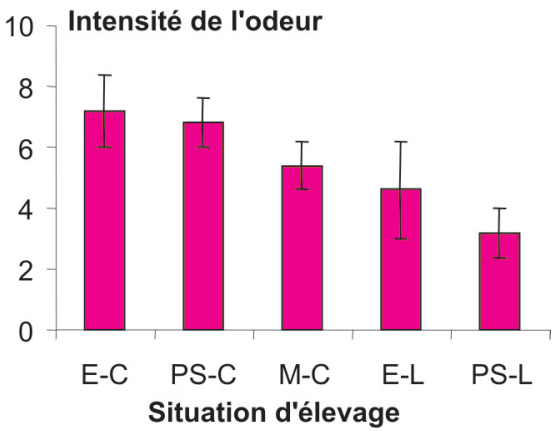

PS : post-sevrage ; E : engraissement ; M maternité ; C : caillebotis ; L : Litière.

\section{2 / Analyses sensorielles}

Les analyses sensorielles ont été conduites en laboratoire par un jury de 10 personnes, notant 9 descripteurs (Porcherie, Fécal, Urine, Chou-Gaz, Couenne, Gras-Rance, Fumier-Etable, Paille, Champignon) et l'intensité globale de l'odeur sur une échelle de 1 (très faible) à 10 (très fort).

La mise en œuvre du piégeage des effluves sur phase lipidique est très simple puisqu'il suffit d'ouvrir la boîte de Pétri et de la positionner à un emplacement déterminé pour piéger les odeurs.

Les descripteurs les plus variables étaient : «Intensité globale» $(\max =6,9$, $\min =1,2)$, «Porcherie» $(\max =5,2$, $\min =0,1)$ et $\langle$ Fécal» $(\max =5,2$, $\min =0$ ). Les autres descripteurs avaient une variance beaucoup plus réduite. L'intensité globale de l'odeur mesurée de façon délocalisée par le jury est bien corrélée à la note d'intensité attribuée in situ par l'expérimentateur (figure 2), ce qui suggère que les analyses sensorielles délocalisées resti-
Figure 2. Relations entre la note moyenne attribuée au descripteur «intensité globale» par le jury d'analyse sensorielle et la note d'intensité odorante attribuée in situ par l'expérimentateur. Les points correspondent aux moyennes et écart-types des notes pour chacune des situations d'élevage (d'après Bégnaud et al 2004a).

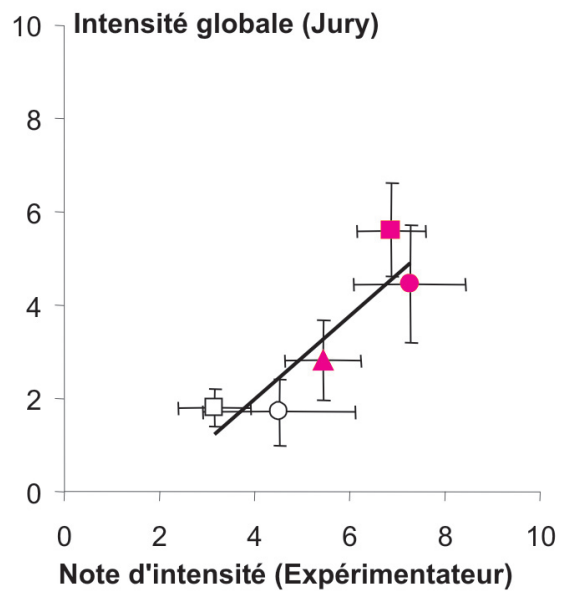

Carrés : post-sevrage ; Ronds : engraissement ; Triangle : maternité ; Rouge : caillebotis ; Blanc : Litière.

tuent bien la variabilité des odeurs perçues sur site.

Une classification hiérarchique ascendante des résultats des 42 élevages conduit à définir trois classes de perceptions olfactives (tableau 1). La classe 1 se distingue nettement des classes 2 et 3 . Elle regroupe 14 situations d'élevages sur caillebotis qui présentent les notes d'odeurs les plus élevées. A l'opposé, les 17 élevages regroupés dans la classe 3 , majoritairement sur litière, ont les odeurs les moins intenses. La classe 2 regroupe 11 élevages, principalement sur caillebotis, avec des caractéristiques d'odeurs intermédiaires.

L'analyse sensorielle délocalisée par flairage en laboratoire des effluents pié- gés sur une phase lipidique permet de séparer les élevages en fonction de la nature du sol sur lequel reposent les animaux (figure 3). L'axe 1 de l'analyse en composantes principales des données sensorielles différencie clairement la majorité des élevages sur caillebotis (odeurs intenses sur site) des élevages sur litière (odeurs faibles sur site). Aucune distinction n'a pu être mise en évidence ni entre caillebotis intégral béton et caillebotis partiel, ni entre litière de paille et litière de sciure de bois. Les salles de maternités, moyennement odorantes, sont proches des élevages sur litière. La variance élevée du plan principal de l'Analyse en Composantes Principales (ACP) (93,9\% dont plus de $90 \%$ pour l'axe 1) reflète l'aspect unidimensionnel des perceptions sensorielles qui se résument à une «intensité globale» décrite par les termes «porcherie» et «fécal» (figure 3).

L'enquête menée sur le terrain a permis d'expliquer la position de plusieurs situations d'élevage qui semblent mal classées dans la description schématique qui vient d'être faite et de confirmer par la même occasion les performances de l'analyse sensorielle délocalisée. Les quatre élevages sur caillebotis se retrouvant en classe 3 présentaient tous sur site une odeur globale très réduite. Il s'agissait de trois salles de maternité particulièrement propres et d'une salle d'engraissement avec une très faible quantité de lisier dans ses fosses lors de l'échantillonnage. A l'inverse, le paillage de l'élevage sur litière situé en classe 2 était de qualité médiocre et odorant.

\section{3 / Analyses instrumentales (SPME-MS)}

Les effluves issus des fibres SPME exposés dans les élevages sont introduits directement dans un spectromètre

Tableau 1. Notes moyennes d'odeur attribuées par le jury d'analyse sensorielle et répartition des situations d'élevage pour chacune des trois classes définies par classification hiérarchique ascendante. Les symboles indiqués pour chaque situation d'élevage renvoient à ceux utilisées dans la figure 2 (d'après Bégnaud et al 2004a).

\begin{tabular}{|c|c|c|c|c|c|c|c|c|c|}
\hline \multirow[b]{2}{*}{ Classe } & \multicolumn{3}{|c|}{ Notes moyenne d'odeur } & \multicolumn{6}{|c|}{ Répartition des situations d'élevage } \\
\hline & $\begin{array}{l}\text { Intensité } \\
\text { globale }\end{array}$ & Porcherie & Fécale & E-C & PS-C & $\mathrm{M}-\mathrm{C}$ & $\begin{array}{c}\mathrm{E}-\mathrm{L} \\
\mathrm{O}\end{array}$ & $\begin{array}{c}\text { PS-L } \\
\square\end{array}$ & Total \\
\hline 1 & 5.6 & 4.0 & 3.8 & 7 & 7 & & & & 14 \\
\hline 2 & 3.5 & 2.3 & 1.8 & 5 & 1 & 4 & 1 & & 11 \\
\hline 3 & 1.8 & 0.3 & 0.1 & 1 & & 3 & 7 & 6 & 17 \\
\hline Total & & & & 13 & 8 & 7 & 8 & 6 & 42 \\
\hline
\end{tabular}


Figure 3. Représentation des situations d'élevage (symboles : cf. tableau 1) et des principaux descripteurs sensoriels sur le plan principal de l'analyse en composantes principales des données de l'analyse sensorielle délocalisée (d'après Bégnaud et al 2004a).

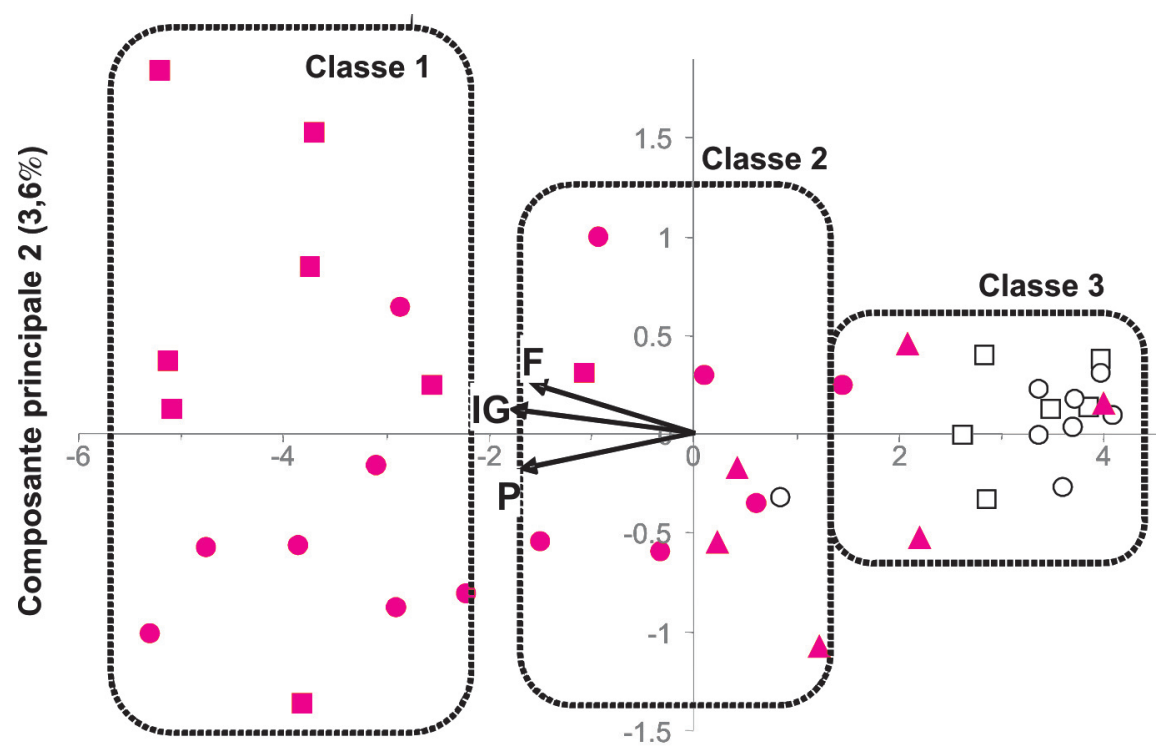

Composante principale $1(90,3 \%)$

F : Fécale ; IG : Intensité globale ; P : Porcherie. de masse (analyse SPME-MS). On obtient ainsi rapidement $(5 \mathrm{~min})$ un spectre de masse qui constitue une signature de l'atmosphère étudiée, bale des Composés Organiques Volatils (COV) qu'elle contient. L'analyse en composantes principales des données de SPME-MS (figure 4) montre que le caractéristique de la composition gloplan principal ne condense que $55 \%$ de la variance des données. Il faut considérer 9 axes canoniques pour en condenser $90 \%$. Cela signifie que l'information disponible dans les empreintes est relativement peu redondante, ce qui est une caractéristique intéressante des spectres SPME-MS. L'observation du plan principal permet de distinguer clairement les situations d'engraissement et de post-sevrage sur litière des
Figure 4. Représentation des situations d'élevage (symboles: cf. tableau 1) et des fragments de masse associés à des composés odorants sur le plan principal de l'analyse en composantes principales des données de l'analyse SPME-MS (d'après Bégnaud et al 2004a).

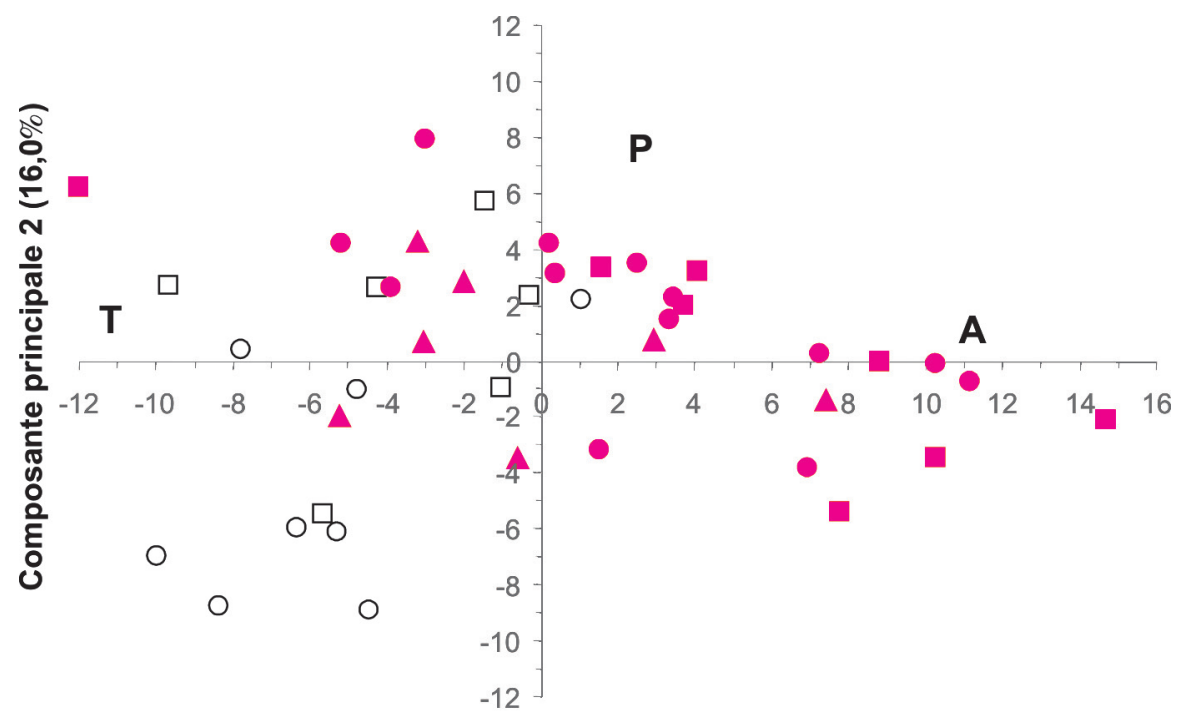

Composante principale $1(38,8 \%)$

$\mathrm{T}$ : terpènes ; $\mathrm{P}$ : p-crésol, 4-ethylphénol ; A : acides carboxyliques et leurs esters éthyliques. situations de post-sevrage sur caillebotis. L'étude des fragments de masse spécifiques de composés impliqués dans les nuisances indique que les situations de post-sevrage sur caillebotis sont associées à de fortes proportions d'ions spécifiques des acides carboxyliques $(\mathrm{m} / \mathrm{z}=60,73$ et 87 : acide acétique, butanoïque, isovalérique et valérique) et des esters éthyliques correspondants $(\mathrm{m} / \mathrm{z}=87,88)$. Les situations d'engraissement sur litière sont caractérisées par les spectres ayant les plus faibles proportions d'ions spécifiques du p-crésol et de l'éthylphénol $(\mathrm{m} / \mathrm{z}=107$ et 108$)$ et des acides carboxyliques, et les plus fortes proportions d'ions caractéristiques des composés terpéniques $(\mathrm{m} / \mathrm{z}=93,121$, 136).

\section{4 / Les analyses instrumenta- les permettent de prévoir les ré- sultats des analyses sensorielles}

Les notes attribuées par le jury d'analyse sensorielle sur les descripteurs «Fécal», «Porcherie» et «Intensité globale» ont été estimées à partir des fragments de masse issus de l'analyse SPME-MS par régression linéaire multivariée. Les données instrumentales permettent de prédire $92 \%$ de la variabilité observée des notes d'intensité globale mesurées par le jury d'analyse sensorielle (figure 5). Elles permettent également de prédire avec une bonne précision les notes attribuées aux descripteurs «Porcherie» $\left(\mathrm{R}^{2}=0,88\right)$ et «Fécal» $\left(\mathrm{R}^{2}=0,91\right)$. Grâce aux analyses séparatives de la composition des

Figure 5. Relations entre les notes d'odeurs estimées à partir des données instrumentales (SPME-MS) et mesurées par le jury d'analyse sensorielle. Les symboles renvoient aux situations d'élevage (cf. tableau 1, d'après Bégnaud et al 2004a)

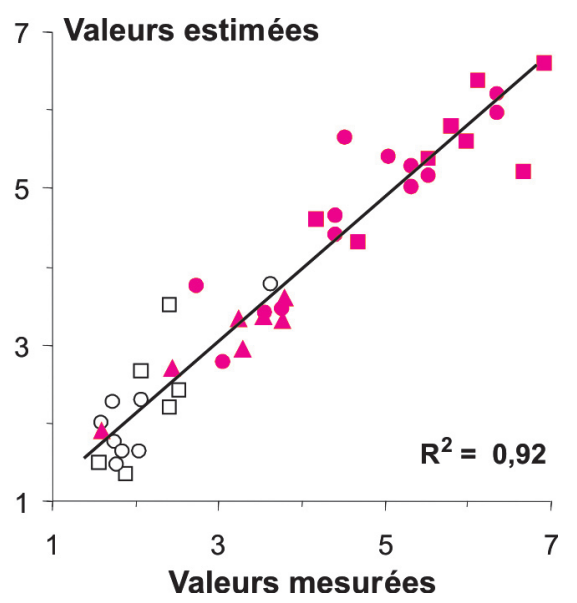


atmosphères précédemment réalisée par Bégnaud et al (2003), il a été possible de remonter aux principaux composés responsables de l'apparition des fragments sélectionnés. Il s'agit de composés connus pour leur contribution à l'odeur des porcheries : composés soufrés, acides gras volatils, indoles, composés aromatiques et furaniques, ce qui montre que l'estimation des caractéristiques sensorielles à partir des données instrumentales est cohérente avec les connaissances actuelles sur les origines moléculaires des nuisances olfactives.

\section{2 / Influence du système de logement sur les odeurs de porcherie}

L'étude présentée au $\S 1$ illustre le fait bien connu que les élevages sur litières sont beaucoup moins odorants que les élevages sur caillebotis (figures 1 et 5). Au sein de ces derniers, les bâtiments abritant des animaux en croissance (post-sevrage, engraissement) émettent plus d'odeurs désagréables que les salles de maternité.

Cet effet bénéfique de la litière a également été observé dans un essai comparant deux situations d'élevage contrastées : l'engraissement sur litière de sciure avec libre accès à une courette extérieure, ou sur caillebotis (Lebret et al 2004, 2006, cf. article sur l'éva- luation globale des systèmes de production). Mesurée à l'aide de la méthode normalisée (AFNOR NF X 43-104 et 43-101), l'intensité d'odeur est beaucoup moins forte dans la salle sur litière que dans la salle sur caillebotis $\left(5,610^{5} \pm 4,510^{5}\right.$ contre $19,010^{5}$ $\pm 13,010^{5}$ unités odeurs par porc et par jour).

\section{Conclusions}

Les avancées méthodologiques obtenues dans le cadre de ce travail semblent ouvrir la voie à la possibilité de mesurer et caractériser les odeurs de porcherie de façon simple et rapide. Par rapport à la méthode normalisée du seuil de dilution olfactif à partir d'un prélèvement d'atmosphère dans une baudruche, la méthode de flairage d'une phase lipidique présente l'avantage d'être plus simple à mettre en œuvre et d'aborder la dimension qualitative des odeurs. Elle n'a cependant été testée que pour des atmosphères relativement concentrées à l'intérieur des porcheries. Sa validité n'est pas établie pour la caractérisation des atmosphères extérieures. L'utilisation de méthodes instrumentales représenterait un progrès décisif en termes d'objectivité et de rapidité de mise en œuvre. Les résultats obtenus avec la technique SPMEMS sont très encourageants mais demandent à être confortés. Il faudra tester la robustesse de la méthode sur des échantillons «externes», indépen- dants de ceux ayant servis à établir les relations entre résultats instrumentaux et sensoriels. Il faudra aussi établir si la méthode est suffisamment sensible pour s'appliquer à des atmosphères extérieures.

Les travaux conduits mettent également sur la voie de composés très malodorants présents en quantité très faibles (données non publiées). De nouvelles recherches seraient nécessaires pour les identifier. Une fois ce but atteint, des travaux pourraient être conduits pour connaître la genèse de ces composés et identifier leurs facteurs de variation.

L'élevage sur litière est un des moyens les plus simples de réduire fortement les nuisances olfactives qui trouvent pour une bonne part leur origine dans les fermentations anaérobies du lisier. Ce mode d'élevage est aussi plus favorable pour le bien-être des animaux (cf. article sur l'évaluation globale des systèmes de production) et il bénéficie d'un a priori favorable de la part du grand public. Le bilan écologique de l'élevage sur litière est pourtant très mitigé (cf. article sur les émissions gazeuses), contrairement à la croyance populaire. La réduction des odeurs peut aussi se faire par une fuite en avant technologique qui consiste à installer des laveurs d'air à la sortie d'évacuations d'air centralisées. Les surcoûts associés à de telles installations sont cependant loin d'être négligeables.

\section{Références}

Bégnaud F., Pérès C., Berdagué J., 2003. Characterisation of volatile effluents of livestock buildings by solid-phase microextraction. Intern. J. Environ. Anal. Chem., 83, 837-849.

Bégnaud F. Pérès C., Murat C., Lebost J, Berdagué J., 2004a. Mise en place de nouvelles méthodes de caractérisation des atmosphères de porcherie. Journ. Rech. Porcine Fr., 36, 39-46.
Bégnaud F., Pérès C., Murat C., Lebost J., Berdagué J., 2004b. Implementation of new methods to characterise atmospheres in piggeries. Int. J. Env. Anal. Chem., 84, 647-659.

Lebret B., Couvreur S., Meunier-Salaün M.C., Guingand N., Robin P., Hassouna M., Cariolet R., Dourmad J.Y., 2004. Comparaison expérimentale de deux conduites d'élevage de porcs en croissance. Journ. Rech. Porcine Fr., 36, 53-62.
Lebret B., Meunier-Salaün M.C., Foury A., Mormède P., Dransfield E., Dourmad J.Y., 2006. Influence of rearing conditions on performance, behavioral and physiological responses of pigs to preslaughter handling, carcass traits and meat quality. J. Anim. Sci., 84, 2436-2447.

\section{Résumé}

Cet article résume les apports du programme «Porcherie verte» dans le développement de méthodes de mesure des nuisances olfactives qui, dans l'esprit du public, sont fortement associées à l'élevage porcin et constituent un facteur limitant essentiel à son développement.

Deux approches, l’une sensorielle, l'autre instrumentale, ont été utilisées conjointement. L'approche sensorielle visait à mettre au point une méthode d'appréciation des odeurs de porcherie qui soit délocalisée (le jury n'a pas besoin de se déplacer sur les lieux), moins lourde que la méthode officielle par dilution d'échantillons d'atmosphères et qui prenne en compte la dimension qualitative des odeurs. Des boîtes de Petri remplies de phase lipidique ont été exposées à l'atmosphère ambiante de porcheries et soumises à une appréciation olfactive par un jury de 10 personnes en laboratoire. Les notations du jury sont bien corrélées à l'appréciation olfactive in situ par la personne ayant réalisé les prélèvements et discriminent bien les situations d'élevage dans l'ordre décroissant d'intensité d'odeurs suivant : post-sevrage et engraissement sur caillebotis $>$ maternité sur caillebotis $>$ litières. 
Pour pallier les inconvénients liés au caractère subjectif de l'appréciation sensorielle, l'approche instrumentale visait à mettre au point une méthode de prélèvement (Micro Extraction en Phase Solide) et d'analyse des composés volatils piégés sur la phase solide (Spectrométrie de Masse). Les signatures spectrales obtenues permettent de différencier les situations d'élevage comme exposé précédemment et de prédire de façon satisfaisante $\left(R^{2}=0,92\right)$ les résultats de l'appréciation olfactive par le jury de laboratoire.

Les avancées méthodologiques obtenues dans le cadre de ce travail semblent ouvrir la voie à la possibilité de mesurer et caractériser les odeurs de porcherie de façon simple et rapide. Ces méthodes n'ont cependant été testées que pour des atmosphères relativement concentrées à l'intérieur des porcheries. Leur validité pour caractériser des atmosphères extérieures reste à établir.

\section{Abstract}

Measure of olfactory nuisance associated with pig breeding

This paper summarises the contribution of the «Porcherie verte» programme in the development of methods that measure olfactory nuisance. Indeed, for the public, olfactory nuisance is associated with pig breeding and is a limiting factor to its development. Two approaches, one sensorial and the other instrumental, were used conjointly. The sensorial approach was aimed at developing a method for appreciating piggery odours that is at a different location (the jury does not have to be on the site), less difficult than the official method using diluted air samples and that considers the quality of the odours. Petri dishes containing lipid phases were exposed to the air in piggeries and were then submitted to olfactory approval by a jury of 10 people from the laboratory. The notes of the jury were well correlated with the in situ olfactory appreciation of the person having taken the samples. The breeding situations are classified in the following decreasing order of intensity: post-weaning and fattening on wood slats>gestation on wood slats>litter.

In order to correct for the inconvenience associated with the subjective character of sensorial analysis, an instrumental approach was used to develop a sampling method (solid phase micro extraction) and analysis of volatile components entrapped in the solid phase (mass spectrometry). The spectra obtained allow the differentiation of the breeding situations as explained above and satisfactorily predict $\left(\mathrm{R}^{2}=0.92\right)$ the results of the olfactory appreciation of a jury from the laboratory.

The methodological improvements made within the framework of this study show that it is possible to measure and characterise odours from piggeries in a very simple and rapid way. However, these methods have only been tested on relatively concentrated air inside piggeries. Their validity for outside air remains to be shown.

BERDAGUÉ J.-L., BONNEAU M., 2008. Mesure des nuisances olfactives associées à l'élevage porcin. INRA Prod. Anim., 21, 361-366. 
